\title{
Imaging prostate cancer
}

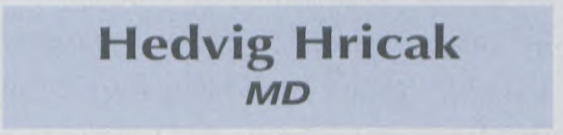

Imaging Prostate Cancer (PCa) continues to represent a clinical challenge. The recommendations range from denial to a strong advocacy for imaging prior to any decision about therapy. Considering the disagreements about prostate cancer detection and choice of treatment, the debate concerning imaging is not surprising.

There are evidence-based guidelines for the use of imaging in assessing the risk of distant spread of PCa. Radionuclide bone scans and computed tomography (CT) supplement clinical and biochemical evaluation (PSA, prostatic acid phosphate) for suspected metastatic disease to bones and lymph nodes. Guidelines for the use of bone scans (in patients with PSA $>10 \mathrm{ng} / \mathrm{ml}$ ) and CT (in patients with PSA $>20 \mathrm{ng} / \mathrm{ml}$ ) have been published and are in clinical use. However while the guidelines are available, due to either lack of communication between specialists, slow dissemination of knowledge or difficulties in changing old habits, the use of imaging has been random and both over and under utilization is often present (CAP CURE survey). CT is an example of over-utilization of imaging, as it is being ordered in $63 \%$ of all newly diagnosed prostate cancer patients regardless of patients' PSA level or
Gleason score. In spite of established guidelines for the use of bone scans there has been no change in the pattern of care. In the last 10 years bone scans continue to be utilized with the same frequency, although in the same time period there has been a significant stage and PSA migration (lower stage lower PSA at the time of diagnosis).

There is no consensus for the use of imaging in the evaluation of prostate cancer local tumor extent. The use of Ultrasound has been limited to biopsy guidance and Brachytherapy seed placements. The use of Magnetic Resonance (MR) imaging has gone through "ups and downs" ranging from enthusiasm to denial. Recent studies indicate that the use of high resolution MRI provides valuable information in the evaluation of local tumor extent, nodal and bony metastases. The combination of MR anatomic information with MR spectroscopic metabolic information is emerging and is being recognized. Another modality offering information about anatomy and metabolism is PET/CT.

The goal for imaging prostate cancer in the next decade is combining anatomy, function and metabolism. Further advances in MR imaging, in MR spectroscopic imaging and other molecular imaging approaches, such as PET/CT and optical imaging will make imaging even more important in the conquest of $\mathrm{PCa}$.

\section{Tumor detection/ localization/ characterization}

Despite the many advances in diagnosis and treatment of prostate cancer, there is still a dearth of information about its biologic potential. At large, this hampers the clinical goal of risk stratification and patient-specific therapy. Knowledge of parameters such as cancer location, aggressiveness, size, and extent, are essential if minimally invasive, patient-specific therapy is to be achieved. TRUS guided prostate biopsy has been considered the standard of reference for tumor localization. Recent reports of biopsy sampling errors and inaccuracies are however troubling. Furthermore, because prostate cancer is a multifocal and histologically heterogeneous disease, biopsy is limited in determining all cancer sites and grades. In fact, when biopsy results were compared with radical prostatectomy for sextant tumor localization, the positive predictive value of biopsy was found to be $83.3 \%$, and the negative predictive value was found to be $36.4 \%$. Magnetic resonance imaging (MRI) and even more so combined MR imaging and proton 3D- MR spectroscopic imaging can be used for patient stratification and targeted biopsy in patients with previously negative biopsies. The addition of metabolic information from 3DMRSI to the morphologic data obtained from MR imaging, leads to a more specific diagnosis and better localization of the cancer than from MR images alone. This approach furthermore allows assessment of tumor aggressiveness. A preliminary 
MRI/MRSI study of 102 patients with step-section pathologic examination has demonstrated a statistically significant $(\mathrm{P}<0.0001)$ correlation between (cancer Choline)/(normal Choline) ratios with the decrease in citrate and elevation of Choline and cancer aggressiveness (Gleason grade).

\section{Staging}

In the evaluation of $\mathrm{PCa}$ local tumor extent, results on the value of TRUS vary widely. Prospective multicenter studies suggest that TRUS is no better than the digital rectal examination in predicting ECE. Endorectal MR imaging offers the best promise for local staging of prostate cancer, but it must resolve problems of reproducible image quality and interobserver variability before it can be recommended for general clinical use. The introduction of MR spectroscopic imaging (MRSI) further expands the value of MR, offering anatomic and metabolic evaluation of PCa. The use of PET/CT at present is reserved for the evaluation of metastatic disease. The introduction of new prostate cancer specific tracers, and the improved localization provided by combined PET/CT technology should present further improved results as well as new applications.

\section{Highlights and practical points for MR and PET technology}

\section{MR imaging}

The signal intensity and detection of PCa depend on the type of imaging sequence used. On T1-weighted images, the prostate demonstrates homogeneous medium signal intensi- ty. On T2-weighted MR images, PCa is shown most commonly with decreased signal intensity within the high-signal-intensity normal peripheral zone. While the detection of $\mathrm{PCa}$ on MRI (similar to transrectal US (TRUS)) has been applicable only to the tumors located in the peripheral zone, recent studies have demonstrated the potential of MR (especially when combined with MRSI) for the evaluation of the tumors in the transition zone as well.

Tumor detection however, may be handicapped by post-biopsy changes. Depending on the time interval between biopsy and the MRI scan, biopsy changes may cause either under- or over-staging of the tumor presence and extent. It has been demonstrated that the MRI study should be performed at least 3 weeks after biopsy. While $\mathrm{PCa}$ detection rates as high as $92 \%$ have been reported, the results of large multi-center studies are disappointingly low, with only $60 \%$ of lesions greater than $5 \mathrm{~mm}$ in any one dimension being detected on MRI scans. The use of MRI in the evaluation of extra-capsular and seminal vesicle tumor invasion is steadily increasing. The MRI findings of extracapsular extension on endorectal coil MRI consist of: an irregular bulge of the gland margin; a contour deformity with a step-off or angulated margin; a breech of the capsule with direct tumor extension; the obliteration of the recto prostatic angle; and asymmetry of the neurovascular bundles. Seminal vesicle invasion is diagnosed when there is: contiguous low signalintensity tumor extension into and around them, and/or when there is tumor extension along the ejaculatory duct. This results in non-visualization of the ejaculatory duct, decreased sig- nal intensity of the seminal vesicles, and decreased conspicuity of seminal vesicle wall on T2-weighted images. While transaxial planes of section are essential in the evaluation of extracapsular invasion, the combination of transaxial and coronal plane images facilitates the diagnosis of extracapsular and seminal vesicle invasion. Variable results have been found for the diagnostic accuracy of MRI in the local staging of PCa. The reported accuracy of MRI in staging PCa ranges from 54 to $90 \%$. These results have raised concerns about interobserver variability and the lack of reproducibility. Over the past 3 years, however, more encouraging results for endorectal MRI have been obtained. The diagnostic performance has improved, with a reported accuracy consistently between 75 and $90 \%$. The recently reported accuracy of MRI in detecting extra-capsular extension $(82 \%)$ and seminal vesicle invasion (97\%), combined with high specificity $(>90 \%)$ in excluding extra-capsular tumors, far exceeds the reported values for either TRUS or CT. The improved performance of endorectal MRI is probably due to the maturation of MRI technology. This includes improvements in MRI technique (e.g. faster imaging sequences, more powerful gradient coils, and post-processing image correction), a better understanding of morphologic criteria used to diagnose extra-capsular disease, and increased reader experience. In the evaluation of lymph node metastases, efficacy data for MRI and CT are similar.

With improvements in MR technology, the role of MRI has extended to every aspect of patient management. It has been shown that the use of endorectal MRI prior to radical 
prostatectomy influences the surgical decision whether to spare or resect the neurovascular bundles. This is especially valuable in high-risk patient groups. In a study reported by Wei $e t$ al. of 76 patients, $24 \%$ had a more aggressive surgical plan when MRI was reviewed together with the clinical examination. In the high-risk group, the Bayesian analysis showed that the probability of needing neurovascular bundle resection increased from $39 \%$ to $78 \%$ with positive MRI findings and decreased from $39 \%$ to $19 \%$ when MRI guidelines were negative. Furthermore, MRI can be applied to predict intraoperative blood-loss during radical retropubic prostatectomy. It has been shown that the prominence of the apical periprostatic veins on MR imaging is associated with greater intra-operative blood-loss during radical prostatectomy. Furthermore, MRI can be used for the prediction of urinary incontinence after radical retropubic prostatectomy. After controlling for age and surgical technique, the multivariant analysis showed that the membranous urethral length is related to the time for achieving stable post-operative continence. A membranous urethra longer than $17 \mathrm{~mm}$ was associated with a shorter time to stable continence.

\section{MR spectroscopic imaging}

The recent developments of MR spectroscopic imaging expand the diagnostic assessment of MR technology beyond anatomic information. MR spectroscopic imaging provides metabolic information specific to the prostate through the detection of the metabolites: Citrate, Creatine, and Choline. This method is based on the fact that the normal gland contains (secretes) citrate, while there is an increased amount of Choline in cancer. This is believed to be due to enhancement of the phospholipid cell membrane turnover associated with tumor cell proliferation, increased cellularity and growth. The method for depicting tumor is based on an increased Choline-Creatine/Citrate ratio.

The information obtained from this new technology allows an improved assessment of tumor location, aggressiveness and the attendant risk of disease progression. In the localization of $\mathrm{PCa}$, positive results from combined MRI and MRS demonstrate a $91 \%$ specificity, which is the highest value obtained by a noninvasive method. The combined use of MR imaging and spectroscopy also significantly improves the evaluation of extracapsular cancer extension and decreases interobserver variability This approach raises further the value of MRI in the evaluation of prostate cancer.

\section{PET/CT imaging}

Most PET/CT studies performed today are diagnostic FDG scans. The basis of cancer detection by FDG is the almost ubiquitous elevation of glucose metabolism by cancer cells. The tumor uptake by FDG, and the resultant value of the test, is cancer site specific. The FDG radiotracer is not well suited for the detection of prostate cancer, especially when the tumor is low-grade. Several alternative tracers are currently under clinical investigation. New tracers are under development showing a promising potential for providing information on tumor biology. One of these tracers is "C-methionine, which differentiates tumor from normal tissue due to elevated protein synthesis. The rapid (10 minute) uptake and plateau of ${ }^{11} \mathrm{C}$-methionine within prostate cancers, allows whole body PET/CT imaging (with decay correction), in spite of the short 20 minute half-life of ${ }^{11} \mathrm{C}$ with minimal interference from the bladder. In search of a non-invasive method to quantify androgen receptors (AR) by PET, ${ }^{18} \mathrm{~F}$-fluorodihydrotestosterone has recently been studied in patients with metastatic prostate cancer. The mismatch between FDG and ${ }^{18} \mathrm{~F}$-FDHT findings suggests the variations in androgen dependence of the different sites. Histologic confirmation of this hypothesis has not yet been performed.

\section{Recommended approach to imaging prostate cancer}

In the staging of $\mathrm{PCa}$, each modality - transrectal US, MRI, CT and $\mathrm{PET} / \mathrm{CT}$ - has advantages and disadvantages. The evaluation by TRUS is restricted to local staging only, while both CT and MRI allow detection of local, nodal, and distant metastatic invasion. The role of CT in staging $\mathrm{PCa}$ is reserved for the search for lymph node metastases, evaluation of advanced disease, and planning radiation therapy. MR offers the most complete evaluation of $\mathrm{PCa}$ assessing loco-regional and nodal disease. The endorectal coil provides higher staging accuracy than the body coil. Discrepancies in the opinion on the value of MR attest to the immaturity and the still developing field of MR imaging. The combination of MR Imaging and Spectroscopic imaging offers anatomic and metabolic infor- 


\section{Gadopentetate dimeglumine}
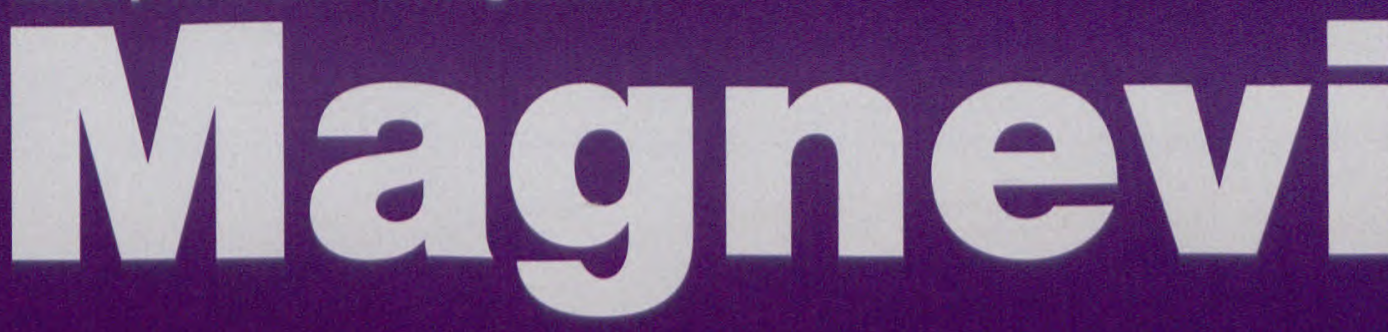

(®) 
mation and appears to be the method of the future for the evaluation of loco-regional disease, while PET/CT, at present is reserved for the evaluation of metastatic disease. Both MR and PET play an important role in the evaluation of treatment response and the future of MR and PET in combination with molecular imaging approaches such as optical imaging can today only be imagined.

In conclusion, improvements, perhaps even revolutions, in the evaluation of prostate cancer are on the horizon, heralded by the many clinical and technological advances, interdisciplinary communication, and critical multicenter clinical trials and outcome studies. Although many questions remain unanswered, and improvements are needed, the great strides that have been made in diagnostic imaging in recent years promise a bright future, getting us one step closer in achieving patient specific, risk adjusted therapy and better outcome for prostate cancer patients.

\section{Suggested reading}

1. Bates TS, Gillatt DA, Cavanagh PM, Speakman M. A comparison of endorectal magnetic resonance imaging and transrectal ultrasonography in the local staging of prostate cancer with histopathological correlation. Br JUrol 1997; 79: 927-932.
2. Coakley FV, Kurhanewicz J, Lu Y, Jones KD, Swanson MM, Chang SD, Carroll PR, Hricak H. Prostate cancer tumor volume: measurement by endorectal MR imaging and MR spectroscopic imaging. Radiology 2002; 223(1): 91 97.

3. Coakley FV, Wei DC, Kattan MW, Eberhardt S Scardino P, Hricak, H. Urinary continence after radical retropubic prostatectomy: relationship to membranous urethral length on preoperative endorectal MR imaging. Urol (in press).

4. Coakley FV, Wei DC, Wasserman ES, Heinze SBJ, Scardino PT, Hricak H. Blood loss during radical retropubic prostatectomy: relationship to morphologic features on preoperative endorectal MR imaging. Urology 2002; 59(6): 884-888.

5. Cornud F. Flam T. Chauveinc L. Hamida K Chretien Y. Vieillefond A. Helenon O, Moreau JF. Extraprostatic spread of clinically localized prostate cancer: factors predictive of p 33 tumor and of positive endorectal MR imaging examination results. Radiology 2002; 224(1): 203-210.

6. Cruz M. Tsuda K. NarumiY. Kuroiwa Y. Nose T Kojima Y. Okuyama A. Takahashi S. Aozasa K. Barentsz JO. Nakamura H. Characterization of low-intensity lesions in the peripheral zone of prostate on pre-biopsy endorectal coil MR imaging. European Radiology 2002; 12(2):357365.

7. Engelbrecht MR. Jager GJ. Severens JL. Patient selection for magnetic resonance imaging of prostate cancer. European Urology 2001; 40(3):300-307.

8. Hricak, H, Wei, DC, Coakley, FV, Reuter, V, Kattan, MW, Gong, MC, Eastham, JA. Scardino PT. Effect of preoperative endorectal MRI on the decision to preserve or resect the neurovascular bundles during radical retropublic prostatectomy. J Urol (in press).

9. Huncharek M, Muscat J. Serum prostate-specific antigen as a predictor of staging abdomi$\mathrm{nal} /$ pelvic computed tomography in newly diagnosed prostate cancer, Abdom Imaging 1996; 21: 364-367.

10. Mueller-Lisse UG. Vigneron DB. Hricak H. Swanson MG. Carroll PR. Bessette A. Scheidler J. Srivastava A. Males RG. Cha I. Kurhanewicz J. Localized prostate cancer: effect of hormone deprivation therapy measured by using combined three-dimensional $1 \mathrm{H} \mathrm{MR}$ spectroscopy and MR imaging: clinicopathologic case-controlled study. Radiology 2001: 221 (2): 380-390.

11. Nutting CM. Corbishley CM. Sanchez-Nieto B.
Cosgrove VP. Webb S. Dearnaley DP. Potential improvements in the therapeutic ratio of prostate cancer irradiation: dose escalation of pathologically identified tumour nodules using intensity modulated radiotherapy. British Journal of Radiology 2002; 75(890): 151-161.

12. Scheidler J, Hricak H, Vigneron DB, et al. Prostate cancer: localization with three-dimensional proton MR spectroscopic imaging - clinicopathologic study. Radiology 1999; 213: 473480.

13. Smith JA, Scardino PT, Resnick MI, Hernandez AD, Rose SC, Egger MI. Transrectal ultrasound versus digital rectal examination for the staging of carcinoma of the prostate: results of a prospective, multi-institutional trial. I Urol 1997; 157: 902-906

14. Tempany, CM, Zhou, $\mathrm{X}$, Zerhouni, EA, et al. Staging of prostate cancer: results of Radiology Diagnostic Oncology Group project comparison of three MR imaging techniques. Radiology 1994; 192: 47-54.

15. Turlakow A. Larson SM. Coakley F. Akhurst T Gonen M. Macapinlac HA. Kelly W. Leibel S. Humm J. Scardino P. Scher H. Hricak H. Local detection of prostate cancer by positron emission tomography with 2-fluorodeoxyglucose: comparison of filtered back projection and iterative reconstruction with segmented attenuation correction. Quarterly Journal of Nuclear Medicine 2001; 45(3): 235-244.

16. Wefer AE, Hricak H, Vigneron DB, et al. Sextant localization of prostate cancer: comparison of sextant biopsy, magnetic resonance imaging and magnetic resonance spectroscopic imaging with step-section histology. I Urol 2000; 164: 400-404.

17. Wolf JS Jr, Cher M, Dall'era M, Presti JC Jr, Hricak H, Carroll PR. The use and accuracy of cross-sectional imaging and fine needle aspiration cytology for detection of pelvic lymph node metastases before radical prostatectomy. $J$ Urol 1995; 153: 993-999.

18. Yu KK, Hricak $H$, Alagappan $R$, et al. Detection of extracapsular extension of prostate carcinoma with endorectal and phased-array coil MR imaging: multivariate feature analysis. Radiology 1997; 202: 206.

19. Yu KK, Scheidler J, Hricak H, et al. Prostate cancer: prediction of extracapsular extension with endorectal MR imaging and three-dimensional proton MR spectroscopic imaging. Radiology 1999; 212: 481-488. 